Cahiers de recherches médiévales

Journal of medieval studies

12 | 2005

La tradition épique, du Moyen Âge au XIX ${ }^{\mathrm{e}}$ siècle

\title{
Le passage à la prose
}

\section{François Suard}

\section{(2) OpenEdition}

Journals

Édition électronique

URL : https://journals.openedition.org/crm/2182

DOI : $10.4000 / \mathrm{crm} .2182$

ISSN : 1955-2424

Éditeur

Honoré Champion

\section{Édition imprimée}

Date de publication : 30 décembre 2005

Pagination : $29-43$

ISSN : 1272-9752

\section{Référence électronique}

François Suard, «Le passage à la prose », Cahiers de recherches médiévales [En ligne], 12 | 2005, mis en ligne le 30 décembre 2008, consulté le 15 décembre 2022. URL : http://journals.openedition.org/crm/ 2182 ; DOI : https://doi.org/10.4000/crm.2182

Ce document a été généré automatiquement le 15 décembre 2022.

Tous droits réservés 


\title{
Le passage à la prose
}

\author{
François Suard
}

1 Depuis son apparition, à la charnière des $\mathrm{XI}^{\mathrm{e}}$ et $\mathrm{XII}^{\mathrm{e}}$ siècles, la chanson de geste n'a cessé d'évoluer. Tout incite à penser qu'il en a été de même avant l'apparition des premiers témoins: le genre épique, attaché à conserver ses perspectives essentielles - la célébration de héros guerriers par la magnification de leurs exploits - a modifié de manière continue ses contenus et sa forme. Il a multiplié les péripéties, donné une place croissante aux épisodes amoureux, fait appel à des motifs et à des schémas de conte populaire; il a abandonné l'assonance pour la rime, le décasyllabe pour l'alexandrin. Nulle transformation pourtant n'est plus importante que l'abandon de la forme poétique (la laisse monorime), puisqu'elle fait disparaitre en quelque sorte les caractéristiques identitaires du texte épique et les frontières qui le distinguent du texte romanesque. De cette transformation, dont la date précise est difficile à fixer, mais qui s'accélère en tout cas après 1450 , nous voudrions aborder quelques aspects : pourquoi a-t-on abandonné le moule épique ? Y a-t-il une écriture commune aux proses épiques? Ces textes ont-ils trouvé un public?

2 Les indications données dans certains prologues des translations en prose sur le projet des auteurs développent essentiellement deux thèmes, celui de l'exemplarité et celui du goût, autrement dit de la mode. Exemplarité par la nature et la qualité du sujet traité : il est question "d'armes, d'amours, de noblesse et de chevalerie $»^{1}$, nous dit le prologue du Roman de Guillaume d'Orange en prose ; or un tel discours convient aux nobles cœurs et aux gens bien nés, comme le souligne Bertault de Villebresme à l'intention de sa commanditaire Marie de Clèves, veuve de Charles d'Orléans, au début de sa mise en prose de la Geste du Chevalier au cygne :

Comme toutes gens de noble et vertueux couraige soient naturellement enclins et curieux de savoir et entendre les haulx gestes et historieulx faiz des anciens roys, princes et seigneurs qui, par leurs magnanimes entreprinses, prudentes conduictes et victorieuses operacions, ont dilaté, espandu et semé leur nom et glorieuse renommee par tous les climatz et angletz du monde, dont il sera memoire pardurable. ${ }^{2}$

3 D. Aubert va plus loin, puisqu'il voit dans les récits qu'il réunit pour les Croniques et conquestes de Charlemaine un modèle à suivre : 
Les fais des anciens doit on voulentiers lyre, ouyr et diligentement retenir, car ilz peuent valoir et donner bon exemple aux hardis en armes et nobles de cuer pour eulx moustrer la fourme et maniere de gouverner leurs corps et noblement contenir, en cheminant le plain chemin ou les patrons de noblesse et parfaitte chevalerie fichierent jadis, en leur vivant, les bournes pour signer la largue et droitte voye d'honneur. ${ }^{3}$

De même, l'auteur de la Chronique associée de Charlemagne et Anséis ${ }^{4}$ considère son récit comme un moyen de «nobles gens esmouvoir a soustenir la foy crestienne et pugner a ses adversaires $»^{5}$. Cependant un tel propos ne doit pas dissimuler la volonté de plaire, à la fois par le contenu et par la forme employée. D. Aubert nuance en effet son propos didactique en évoquant le plaisir que procure une telle lecture :

Si s'en resjouissent encoires et recreent les creatures humaines, et y oublient leurs fantasieuses ymaginations, dechassent et comme vent envoient ensus d'eulx ${ }^{6}$ les temptations dont huiseuse degaste ceulx qui le veulent ensieuvir. Et le gracieux plaisir de la joyeuse lecture ou ilz se delictent, rend leurs esperitz fiers, legiers, joyeux et en eulx mesmes bien disposez, ou aultre passe temps leur feroit dommage et aporteroit ennuy et merancolie ${ }^{7}$.

5 Il semble y avoir en effet un goût pour les récits issus de la chanson de geste, goût indissociable de la prose :

Plus volentiers s'i esbat l'en maintenant qu'on ne souloit, et plus est le laingage plaisant prose que rime, ce dient ceulx aux quieulx il plaist et qui ainsi le veulent avoir,

affirme encore l'auteur du Guillaume, tandis que l'auteur de la Chronique associée justifie l'usage de la prose comme étant « a l'appetit et cours du temps».

7 Avant d'aller plus loin, analysons un peu ces différentes affirmations. Pourquoi la prose, et quel profit espère-t-on de telles lectures? On peut évidemment répondre de façon distincte aux deux questions. Pour la prose, on invoquera la modernisation souvent indispensable d'une langue qui n'était plus comprise et l'avantage d'une forme qui paraît plus usuelle que celle du mètre pour des ensembles narratifs, les romans arthuriens en prose ayant montré la voie dès le XIII siècle. Bertault de Villebresme, qui écrit après 1465, met l'accent sur la difficulté qu'offre à la compréhension le modèle suivi : le livre sur lequel il travaille, dit-il, est «en ancienne rime et asséz obscur lengaige difficille a compregnoir et entendre "; obéissant au commandement de sa dame, il veut donc « mectre et translater icellui livre et memoriable histoire de ladicte ancienne rime et obscur lengaige en prose et lengaige françois cler et entendible », le livre en question étant la version Beatrix des Enfances du Chevalier au Cygne, du Chevalier au Cygne et de la Fin d'Elias 8 .

8 En ce qui concerne les sujets traités, on invoquera à juste titre le rêve chevaleresque des $\mathrm{XIV}^{\mathrm{e}}$ et $\mathrm{XV}^{\mathrm{e}}$ siècles, dont l'exercice de la guerre, tel que nous le rapporte Froissart dans ses Chroniques ou, plus tard, le Maréchal de Boucicaut, souligne l'importance, ou que la cour de Bourgogne, dans son souci de se présenter comme l'héritière de l'empire de Charlemagne et la fédératrice de la lutte contre les Infidèles, a revendiqué comme son patrimoine. Le prologue d'un récit chevaleresque directement écrit en prose dans le milieu bourguignon, mais qui reprend les grands thèmes des proses épiques, Les Trois Fils de Rois ${ }^{9}$, est particulièrement intéressant à cet égard. Après avoir évoqué d'une manière générale l'importance de l'exemple des anciens, l'auteur poursuit :

Et pour ce que la jenne chevallerie a tousjours bon besoing de soy exerser en fais vertueulx, ceulx quy plus ont veu et retenu font, pour les endoctriner, ramener a leur memoire les fais vertueulz des nobles et puissans princes jadiz regnans, en 
racomptant de leurs prosperitez, adversitez, bonnes et mauvaises fortunes et adventures, et comment, par leur haulte conduitte et dilligence, ilz ont desconfiz au trenchant de l'espee leurs mortelz ennemiz. ${ }^{10}$

partir de 1450 et leur succès dans l'édition, associer le phénomène prose et le goût pour
un domaine narratif qui associe, comme le prétend et le fait l'auteur du Guillaume, armes, amour, noblesse et chevalerie.

11 Armes : les proses sont en effet fertiles en combats, tout particulièrement en combats livrés contre les adversaires de la foi. Comme on sait, l'intérêt pour la croisade ne disparaît pas avec la fin des expéditions effectivement menées outremer ${ }^{11}$; sans doute le Turc remplace-t-il progressivement le Sarrasin, mais il s'agit toujours de l'infidèle et de l'imaginaire d'une chrétienté victorieuse de celui-ci.

12 Amour. Très tôt - dès la Prise d'Orange des années 1150 - la conquête amoureuse a pu figurer comme l'un des thèmes de la chanson de geste, associé à la conquête guerrière. Sans doute, dans ce texte légèrement parodique, le message est-il quelque peu brouillé, puisque c'est Guillaume lui-même qui est vaincu par l'amour, avant la belle Orable ; mais ailleurs, dans Beuves de Hantonne par exemple, c'est une belle étrangère, parfois sarrasine, qui est séduite par le charme irrésistible du héros. Les textes ultérieurs auront tendance à multiplier les conquêtes amoureuses du chevalier chrétien ${ }^{12}$, et les proses leur emboîteront le pas.

13 Noblesse. Les héros appartiennent à la plus haute noblesse et sont fréquemment alliés au souverain. Dans le même temps, par les vicissitudes qui entourent les débuts du héros dans les récits d'enfances, vicissitudes dont ils triomphent par leur courage et leur détermination, ils constituent des exemples dans lesquels, sans doute, les lecteurs non nobles peuvent se reconnaître.

14 Chevalerie. Le terme n'ajoute probablement pas aux autres, il les résume plutôt, grâce à sa valeur polysémique. Fait en effet partie de l'univers chevaleresque celui qui accomplit des exploits (armes), qui est amoureux et appartient à la noblesse. Chevalerie permet peut-être aussi de rendre plus attractif le récit issu de la chanson de geste en projetant sur lui l'aura du roman d'aventures chevaleresques tels que le Baudouin de Flandres, le Comte d'Artois ou Robert le Diable.

15 Ainsi la prose-type du $\mathrm{XV}^{\mathrm{e}}$ siècle apparaît-elle comme un récit à l'intérêt varié, dépassant le "schématisme" de la chanson de geste, mais qui, à la différence des romans chevaleresques, invite à considérer l'histoire proposée et ses héros comme une fresque historique dont l'exemplarité tient au caractère véridique des faits rapportés : le lecteur peut s'identifier aux héros comme à de véritables ancêtres.

Les choses, pourtant, ne sont pas si simples, et l'on pourra distinguer, à l'intérieur des proses épiques, grâce à l'étude du style, des tendances assez différentes, qui vont d'une

Cahiers de recherches médiévales, 12 | 2005 
écriture rapide, tendant à l'abrègement, à la recherche d'un style orné et développé, ralentissant l'action mais approfondissant l'analyse des personnages et des situations.

Une comparaison entre la façon dont la prose de l'Arsenal 3346 des Loherains et celle de David Aubert rendent compte du meurtre de Bègue de Belin par les forestiers peut être éclairante à cet égard. La prose de l'Arsenal s'en tient à la succession des faits, qu'elle résume en quelques lignes, faisant disparaître tout repère par rapport à la succession des laisses et omettant tout discours rapporté :

Et quant ilz vindrent la ou estoit Beggon, le bon chevalier, ilz le crierent et luy dirent que il lui failloit morir car il avoit robé la forest Fromont. Beggon leur dist que il n'estoit pas larron et que il estoit chevalier et, c'il avoit fait riens mal a point, que il estoit prés de l'amender audit Fromont et ses chevaliers. Le fourester ne fut contant, si soy traict prés Beggon et luy voulsist ouster son cor. Beggon soy corroucza car il n'avoit pas a coustume par tielx gens estre mal mené, si le frappe $\mathrm{du}$ poign seur le coul et le tue. Et tantost les autres .VI. le vindrent envaïr. Lors, qui vist Beggon bien ferir adés a l'un et puis a l'autre, bien dit on que il estoit chevalier, et si bien se deffendit que il en tua troys. Et les autres trois s'en vouloient fouir, quant ilz virent ung autre fourester qui leur vint a l'aide, qui estoit bon archier et $\mathrm{i}$ avoit arc et trousse. Cest archier ferit Beggon d'une fleche par mi le corps si fort que la mourut. ${ }^{13}$

Si l'on compare avec la chanson de Garin le Loherain ${ }^{14}$, on constate l'importante contraction du texte: 14 lignes dans l'édition Herbin contre 105 vers. Les étapes successives de l'action sont reprises dans leur enchaînement, sauf l'ultime exploit de Bégon, qui consiste, alors qu'il est frappé à mort, à percer le meurtrier de son épieu ; mais certains éléments de caractérisation manquent, comme le lien de parenté qui unit l'archer au forestier tué par Bégon au début de l'action: le premier est le neveu du second. Les reprises caractéristiques d'une laisse à l'autre ont disparu; toutefois une formule, reprise dans trois laisses successives de la chanson (" qui dont veïst » a laissé une trace ("qui vist Beggon bien ferir»). Le prosateur s'intéresse non seulement aux faits eux-mêmes, mais à leurs causes: il indique brièvement les motivations des personnages (le forestier qui ne se satisfait pas des explications de Bégon; le fait que le héros ne fraie pas avec les vilains). Le résultat est une relation concise des événements, qui a pour but de les consigner et de les proposer à la mémoire du lecteur; dans son dépouillement relatif, elle ne manque pas de puissance dramatique, montrant la brutalité et l'absurdité de la mort d'un héros confondu avec un braconnier.

Le texte de David Aubert est d'une tout autre nature. Dans l'édition de Valérie Naudet, ${ }^{15}$ il occupe 61 lignes, ce qui est du même ordre que les 105 décasyllabes du modèle. Le récit suit pas à pas le modèle en vers mais le transforme par une procédure complexe qui vise à construire un récit majestueux, à la fois dramatisé et vraisemblable. On notera tout d'abord la concentration des discours rapportés. La première intervention de Thibaut du Plessis, allié de Fromont, qui est le chef de la troupe des agresseurs et l'exhorte à s'emparer de Bégon (v. 9889-92) est remplacée par un discours au style indirect, attribué à l'ensemble du groupe ("ilz parlerent l'un a l'autre », 1. 304. La scène s'en trouve dramatisée, car elle oppose tout de suite une meute au héros solitaire.

Par ailleurs les échanges entre les protagonistes créent, peut-être à la mesure du retardement qu'ils introduisent, une tension efficace. D'abord vient l'apostrophe des veneurs, les « sept compaignons » qui, en une longue période, menacent celui qui a osé offenser les propriétaires de la forêt et, plus qu'eux tous, Fromont. Ensuite, au lieu de donner immédiatement la réponse de Bégon, le narrateur ménage un habile suspens en analysant l'état d'esprit du héros, qu'il montre serein en dépit des mises en garde 
inquiétantes de son épouse, qu'il est seul à rappeler ici $^{16}$. Aucune indication sur ce que pense Bégon dans le texte en vers, mais le ton est celui d'un homme soucieux de se faire respecter tout en étant décidé à vendre chèrement sa vie : il se déclare prêt, s'il le faut, à faire amende honorable, mais un aparté le montre prêt au combat :

Puis dit aprés : « Or ai dit que laniers !

Dex me confonde qui tot a a jugier

se je m'i rant por ses .vii. pautoniers!

Ainz qu'il m'ocient, me cuit je vendre chier $!{ }^{17}$

La réponse de Bégon, chez David Aubert, ne reprend que l'acceptation, faite " courtoisement ", d'une amende honorable, après quoi les échanges entre le héros et les veneurs, qui dans la l. CIV sont au style direct, sont pris en charge par le narrateur au style indirect et, au lieu de continuer à s'inscrire dans une perspective théâtrale, soulignent la dimension narrative du passage. Il n'y aura plus dans la suite de discours rapportés - alors que, devant les hauts faits de Bégon, les échanges entre les agresseurs survivants, notamment Thibaut, sont nombreux dans la chanson - sauf l'injonction adressée au parent du forestier, passage retenu peut-être parce qu'il prépare immédiatement la mort du héros.

Un autre élément de dramatisation consiste dans l'étude et la caractérisation des personnages, comme pour le futur meurtrier du héros. Ce dernier n'est qu'un auxiliaire ( « ung villain garchon ${{ }^{18}}^{18}$ ), mais il est présenté - ce qui ne figure pas dans la chanson comme le fils, et non le neveu, du forestier qu'il est en train de chercher : «Et quy demanderoit que celluy garchon aloit querant et quy il estoit, dist l'istoire que il estoit filz du forestier et que il le cerchoit ça et la par la forest » (1. 344-46). L'attente du jeune homme et la relation familiale qui l'unit au forestier rendent donc encore plus plausible que dans la chanson le fait qu'il obéisse sans hésiter à l'ordre qui lui est donné, ordre lui-même plus ramassé et plus efficace que dans le modèle: "Va tost et tu le [le forestier] trouveras et celluy qui l'a occis dessoubz ung hault tramble que la peulz veoir $»^{19}$

Ajoutons l'addition de certains détails qui accroissent l'illusion du réel ${ }^{20}$, une écriture ample mais non emphatique, et nous pouvons reconnaître dans la prose de D. Aubert un roman d'aventures dramatiques bien construit, où les motivations des personnages sont précises.

Lorsqu'un même sujet est traité par deux proses différentes, des différences d'écriture peuvent être relevées, qui témoignent chez les auteurs de conceptions et de styles différents. L'exécution du fameux gab d'olivier dans Galien est contée rondement dans le ms. 1470 de la BNF, édité par H.-E. Keller :

Et ainsi commanda le roy Hugues a appareiller ung lit pour coucher sa fille, le quel fut moult richement paré. Or fut elle couchee, et Olivier auprés d'elle, au quel il tardoit moult de la tenir entre ses braz. Si l'embrassa et baisa tout a son plaisir et tant fist que .xii. fois lui fist la besoigne avant qu'il fust temps de lever. Lors dist Olivier a la belle: «M'amie, il nous convient dormir entre vous et moy. - Certes, dist la belle, mon amy, j'en suis bien contente. » Si s'embrasserent l'un l'autre et s'endormirent jusques a tant qu'il fust grant jour, tant que Olivier s'esveilla, qui fut tout esbahy. Si s'esveilla la belle, et puis la baisa tant de fois qu'il lui pleut et puys monta dessus, et au descendre se trouva moult lassée ${ }^{21}$.

Bien que la matière soit identique, le récit est plus ample, plus orné dans la prose du ms. Arsenal 3351 (la Geste de Monglane) et porte la trace de réticences du scripteur - à moins qu'il ne s'agisse d'un procédé d'appel : 
Et adont fut ung lit commandé a drecier noblement et si richement comme a fille de roy appertenoit, et finablement furent couchié celle nuit ensamble, qui fut cose moult mervilleuse a faire et acorder, mais a croire plus. Si le tesmongne l'istoire, laquelle je ne puis mie desdire, car elle dit que en celle nuit se contint et porta avecq elle le conte Olivier si amoureusement que .xii. fois s'aquita naturellement envers elle et non plus, car le jour aprochoit, et nature, qui le repos du corps desiroit, lui failly au besoing, si qu'il le convint rendre aux armes et mercy prier a la damoiselle, laquelle fut cause de sa salvacion, comme l'istoire le recordera.

Dieux, comme furent en grant consolacion Olivier et Jaqueline, la fille au roy Huguon! L'un acola l'autre par grant amytié quant .xii. fois se furent ensamble esbatus, mais tant y eust qu'ilz s'endormirent bras a bras si doulcement que le jour parut grant et cler sur eulx, si que Olivier, qui premier s'esveilla, fut plus dolant que ne pouroit recorder l'istoire, et non sans cause, car sa vie y pendoit, comme tout asseuré en estoit ${ }^{22}$. elles sont patentes. D'une part le narrateur, qui se retranche derrière sa source, se déclare peu convaincu par cette nuit exceptionnelle, ce qui revient à souligner que le gab d'olivier et son acceptation par le père de la jeune fille lui semblent étonnants, voire choquants. D'autre part le récit de cette nuit est conté sur un registre plus élégant: «s'aquita naturellement » remplace le plus trivial « lui fist la besoigne » ou «monta dessus» du Galien, qui ajoute d'ailleurs une reprise aux douze étreintes auxquelles s'arrête le narrateur de la Geste de Monglane : «nature, qui le repos desiroit, lui failly au besoing » développe "se trouva moult lassé ». La description du sommeil gracieux des amants remplace un bref échange de répliques, et l'on peut considérer en somme que le prosateur de l'Arsenal travaille à sauver de la grivoiserie une scène qui l'étonne - ou dont il pense qu'elle étonnera ses lecteurs - alors que celui du Galien traite son sujet avec bonhomie et alacrité.

Il y a donc différentes façons d'écrire la prose épique, et les éditions récentes, qui font généralement précéder le texte d'une comparaison entre la prose et le vers, le montrent bien. Piotr Tylus, éditeur de l'Histoire de la reine Berthe et du roy Pepin ${ }^{23}$, ne consacre pas moins de 48 pages à l'étude du «Processus de la mise en prose ${ }^{24}$ et relève par exemple la subsistance de certaines traces de l'écriture épique: «les débuts de paragraphes qui ont la valeur d'intonations épiques et les fins de paragraphes avec la valeur conclusive $\aleph^{25}$; Michel J. Raby, dans son édition de Huon de Bordeaux en prose ${ }^{26}$, propose une étude encore plus développée (pp. LIII-CxLIV), dans laquelle il signale par exemple

les expansions à caractère psychologique. Celles-ci, à valeur dramatique et qui se placent aussi sous ce même signe de la précision, sont pour la plupart sous la forme de brefs portraits, petites scènes, récits ou monologues. Le remanieur les utilise soit pour rehausser ou créer le pathétique ou le comique d'une scène ou d'un personnage, soit pour mettre en valeur l'exploit, soit, enfin, indirectement comme faire-valoir ou comme tremplin dramatique ${ }^{27}$

Mais de telles études présentent l'inconvénient de tirer leurs enseignements de la seule confrontation entre la prose et son modèle en vers; pour mesurer la diversité des procédures, il conviendrait de comparer entre elles plusieurs proses suivant des critères précis (par exemple attaque des paragraphes, présence des périodes et des ensemble synonymiques, usage du style direct, comparaisons, portraits, analyses psychologiques) puis de croiser ensuite ces données pour dégager diverses manières d'écrire.

Cahiers de recherches médiévales, 12 | 2005 

l'Histoire de la reine Berthe et Huon de Bordeaulx, les différences sautent aux yeux. Pas de division en chapitres dans le premier texte, mais une succession de paragraphes aux incipits variés : indications temporelles («Ung jour d'esté », 1. 29 ; Auffort, 1. 179 ; « Un jour », 1. 191 ; « Asses paroles », 1. 349 ; « Lors », 1. 479, etc.) ; exclamations invocatives : «Saincte Marie » (cinq fois ${ }^{28}$ ), « Dieulx » (onze fois) ; mise en évidence d'un nom propre (Berthe, Pépin, etc.) ou du nom commun qui le remplace («le roy», «la royne »...) : 96 fois pour 135 paragraphes.

Huon répartit au contraire sa matière en chapitres de longueur variée, mais dont la caractéristique majeure est le très petit nombre de paragraphes et le recours quasi exclusif à une indication temporelle pour les attaques. Plusieurs chapitres ne comportent qu'un seul paragraphe : 1. 5-22, 25-70, 130-163, 423-523, 651-747, 857-888, 892-934. De plus, si l'on considère les trois derniers exemples cités, on voit qu'un paragraphe unique peut convenir à des portions de récit de dimensions variées $(100,97$ et 30 lignes $^{29}$ ).

31 En ce qui concerne les incipit de chapitre, les indications temporelles et notamment la subordonnée introduite par quant se taillent dans le même texte la part du lion, la même formule pouvant être répétée au cours du même chapitre. Ainsi, pour le chapitre qui commence à la ligne 1532, on trouve successivement "Quant Huon et Garin furent entrez. », "Quant Huon fut descendu» (1.1613), "Quant Huon eut ouy parler.» $(\text { l. 1669) })^{30}$. D'autres embrayeurs temporels coexistent avec quant: « alors » (1l. 76, 572, 789, 3493, 3585), «lors » (1. 544), « alors que » (1l. 379, 1225, 1785), « aprés que » (892), « aprés ce que » $(11.1332,2213,2308,6081,7039)$, parfois le redoublent (« alors, quant », 1. 3081). Il existe d'autres embrayeurs, mais moins fréquemment employés : quelques formules analeptiques en tête de chapitre : «Bien avez ouy » $(1.332,2611,3008,5319)$, «Bien avez entendu» (1. 6716) « ainsy comme vous avez ouy» (1. 937, 3883), « ainsi comme cy devant avez ouy» (1.3515) «bien avez ouy par cy devant» $(1.3559,4530)$, «Par cy devant avez ouy comment» (l.1392), «Ainsi comme vous oyez» (l.6181), «Ainsi que les deulx freres se devisoyent (1.6284). On trouve aussi des formules de référence au conte suivi : "Or dit le compte» $(1.3788,4462)$, «Or dist l'hystoire » (1. 5459), «Or deit nostre ystoire » (1.4616) des consécutives à valeur temporelle (« tant chevaucha Huon qu'il vint», 1.996). L'ouverture par le discours direct est exceptionnelle : nous n'en avons repéré qu'un seul exemple, à la 1. 6599 (« Sire, ce deist Gerard»).

le voit, le prosateur de Huon est au fond indifférent au paragraphe, dont l'étendue peut coïncider avec celle du chapitre. Il entend souligner grâce aux indications temporelles l'enchaînement des chapitres, et c'est à l'intérieur de ceux-ci que des articulations variées, mais très souvent temporelles elles aussi, bâtissent la structure interne du récit. L'examen du très long chapitre racontant, à la fin du roman, le jugement de Huon par Charlemagne à Bordeaux (1.6716-6999) est éclairant à cet égard et montre les paragraphes possibles.

Par une formule analeptique («Bien avez entendu»), le début du chapitre renvoie à la décision prise par Charlemagne d'aller à Bordeaux puis conte les préparatifs du départ : il se termine par la prière adressée par le narrateur à Dieu pour qu'il protège Huon, et une formule de liaison introduit ce qui pourrait être un second paragraphe : "Ainsi comme je vous dy, le roy Charlemaigne. » (1.6727-28). Un deuxième paragraphe peut être constitué par le récit de l'arrivée à Bordeaux et le dialogue entre le héros et le

Cahiers de recherches médiévales, 12 | 2005 
méchant geôlier qui lui annonce sa mort prochaine. En tout cas, à la 1.6752, une nouvelle formule ("Ainsi comme vous oyez») montre la clôture de ce dialogue et introduit la scène de banquet violemment interrompue par Naymes. Le récit est ensuite scandé par une succession d'alors, dont le premier introduit l'arrivée de Huon (1. 6774), le second les préliminaires à son plaidoyer (1. 6796, et le troisième la première partie de ce plaidoyer (1.6811). La défense du héros, qui constitue un très long discours (1.6797-6944), comporte elle-même plusieurs articulations, qui pourraient délimiter autant de paragraphes. Une première partie prend fin à la 1.6853 , après que Huon a évoqué son mariage et désigné Esclarmonde à la compassion des barons; une temporelle introduit le mouvement de pitié des assistants ("Quant les barons.»). Les autres parties sont structurées par les interventions de Naymes, qui approuve le propos du héros («Huon, ce deist le duc Naymes...», 1.6883), puis le geste de Gérasme, qui montre ses blessures ("Alors Gerasmes se mist avant », 1.6922) et ouvre un dialogue à plusieurs voix au terme duquel Charles décide de «mener par jugement » Huon; une temporelle ("Quant les barons... », 1.6973) ouvre la dernière partie qui scande ellemême par une série de temporelles les préparatifs et le début de la délibération ("Adonc Huon... », 1. 6980 ; « Alors le roy Charlemaigne. . », 1. 6981 ; « Quant les pers... », 1. 6987 ; « Quant le duc Naymes... », 1. 6992).

La renonciation aux paragraphes, traces probables des laisses dans Berthe, ne signifie donc pas ici dilution de la structure narrative, mais plutôt recherche d'une continuité qui n'exclut pas une délimitation relativement claire des différentes parties du récit. Quant à la structure et au développement de la phrase, elle peut varier d'une prose à l'autre comme à l'intérieur d'une prose déterminée. M. Raby, analysant la phrase dans Huon, la présente ainsi :

Tour à tour longue, aux contours mal définis, sans rythme harmonieux (conséquence de cette aspiration constante à trop vouloir dire), ou au contraire, courte, d'aspect inachevé et d'allure saccadée, elle semble être le résultat des fonctions antithétiques inhérentes à certains de ses éléments constitutifs: subordonnants, adverbes ou conjonctions qui les lient et les articulent ${ }^{31}$.

Le jugement esthétique est peut-être un peu sévère, mais l'analyse est exacte, comme le montre le passage suivant, situé au début du voyage de Huon vers Babylone :

Huon print congié de luy tout en plourant et se partit et ce mist en la riviere du Tibre sur une moult riche nef, laquelle le Sainct Pere luy avoit fait apareiller, moult bien garnie de tout ce que mestier luy estoyt. Atant monta en la nef. Il eust bon vent. Tost fust a Brandis, mais ains que il fust la arrivé, il ploura maintes larmes, et mainct pyteux regrect fist de ce que ainsi le convenoyt departir de son pays. Mais les gens le resconfortoyent en luy disant mainte belle exemple pour le resconforter ${ }^{32}$.

Effectivement, après la première phrase, qui comporte plusieurs propositions, les deux indépendantes très brèves, qui précèdent deux phrases un peu plus développées, surprennent. Mais on peut se demander si l'imprimeur a correctement composé le modèle qu'il avait sous les yeux : il suffirait en effet de mettre un ou (=où) entre les deux indépendantes pour atténuer "l'allure saccadée ", et de relier par une simple virgule les phrases 4 et 5 - ce qui paraît de toute façon souhaitable -, pour obtenir une seconde période, plus ample que la première. En était-il ainsi dans le modèle, et l'imprimeur a-til brisé, volontairement ou non, un rythme trop majestueux? Nous n'en savons évidemment rien, puisque le manuscrit de la prose a disparu. 
L'alternance entre phrases longues et brèves se rencontre ailleurs, au moins dans les parties narratives de la prose, comme on le voit dans le Fierabras de Jehan Bagnyon; avec lui, suivons le traître Marpin qui se prépare à entrer dans la tour où sont réfugiés Floripas et les chrétiens :

Et sur ce, quant il fut bien vespre, secretement s'en entra és fossés, qui estoient plains d'eaue, et passa oultre. Puis, quant il fut au pié de la tour, a ses engins subtilz moult legierement il monta és fenestres et entre en la tour et allume la chandoille. Et puis il entra en la chambre de Florippes et la trouva serree, mais a faulces parolles dyaboliques il la fist ouvrir. Et quant il fut dedens, il va veoir les barons, qui estoient endormis, et fist ses enchantemens que pour nulle riens ne se puissent esveiller. Et puis vint au lit de Florippes et sercha secretement tant qu'il trouva la centure, et la ceint autour de luy ${ }^{33}$.

Ici, on le voit, le scripteur semble vouloir faire alterner phrases courtes et phrases plus longues, mais d'ampleur toutefois limitée. D'autres prosateurs cherchent visiblement à polir leur style, comme nous l'avons vu avec le Garin de D. Aubert ou le Roman de Guillaume d'Orange, dont nous avons autrefois souligné le caractère majestueux. Chez lui, même les scènes d'action ne semblent pas conduire à accélérer le rythme, à raccourcir le souffle. Ainsi, tandis qu'Aimery fait une sortie hors de Narbonne, le Sarrasin Thouloumer l'observe :

Thouloumer d'Inde, qui l'une des batailles avoit en sa commende, veant l'outraige que Aymery avoit fait d'avoir sa cité ainsi seule laissee et habandonnee, se tira de la presse lors et, pour tendre affin que on meist peinne d'avoir la cité par assault, par emblee ou aultrement, se departi le plus hastivement qu'il peust et fist tant qu'il trouva l'amiral, auquel il dist: «A cestui coup avrés vous Nerbonne ou jamais, sire amiral, fet il, car de la s'en est Aymery sailli atout ce qu'il avoit de gens. Et tandis qu'il festoiera ses enffans, qu'il ne vist long temps a, pourrés vous envoyer, et ce pendant tendrés estal cy endroit et garderés la baniere Mahom, a ce que les Crestiens n'en puissent issir. $\|^{34}$

Examinons maintenant, du point de vue de l'épique, le résultat des transformations dues à la prose. Le lien essentiel entre lyrisme et récit se trouve rompu de façon structurelle avec la disparition de la forme versifiée, même si une telle rupture existait déjà dans les faits avec la prévalence du narratif dans les chansons de geste du $\mathrm{XIV}^{\mathrm{e}}$ siècle. Ce n'est pas que les proses soient en elles-mêmes incapables de lyrisme : Beuves de Hantonne présente d'assez nombreux passages réussis à cet égard ${ }^{35}$, et le Guillaume lui-même laisse parfois une place à l'émotion, comme lorsque Gérard de Commarchis, à Barbastre, croit devoir bientôt être séparé de son amie Sallatrie :

« Adieu, noble païs de France, fet il, ou jamais ne retourneray comme je voy; et sy ne verray jamais amy ne parent que je aye puis que failli avons a leurs secours, ainçois me lesseray octire, car jour de ma vie ne me acorderay a ce que Beuffvon le mien pere rende ceste cité par tel qu'il me conviengne m'amye laissier $»^{36}$.

40 Mais un tel lyrisme, savamment maîtrisé, cherchant dans la prose rythme et harmonie grâce à l'équilibre des séries syntaxiques, est dissocié du récit et se rencontre surtout dans les passages où s'exprime le sentiment de l'amour. Dans la chanson de geste au contraire, même si la plainte amoureuse trouve dans certains textes un espace accru et spécifique (Beuves de Hantonne), l'action, et l'action guerrière en particulier, reste en symbiose, du fait du recours aux procédés habituels de la chanson de geste, avec le lyrisme. Beuves de Hantonne, à cet égard au moins, reste pleinement une œuvre épîque.

41 La dissociation réalisée dans les proses ne se trouvera pas atténuée, bien au contraire, lorsque certains textes tardifs comme La Conqueste de Trebizonde ou la prose du ms. BNF 
fr. 12791 insèreront dans leur récit plusieurs passages versifiés. Introduire un rondeau, une ballade, une complainte dans un récit en prose revient à souligner la rupture entre les deux formes choisies, le passage versifié ne servant que d'ornement, de parure à un texte qui poursuit une voie différente. Ainsi, dans le ms. 12791, lorsque les princes chrétiens donnent leur accord pour partir en Espagne et lutter contre Fierabras et chargent Regnier de Gennes de transmettre leur réponse à Charlemagne, celle-ci prend la forme d'un rondeau se détachant nécessairement du contexte :

Chef triumphant, magnifique noblesse,

Imperateur precellant en haultesse,

Prince puissant, fonteyne d'equité,

A vous servir prenons felicité:

Vostre regnon nous acroist hardiesse.

Le fier Ballant que la noblesse blesse

Sera destruict et mis en tel detresse

Qu'a tousjours mais sera desherité.

Chef triumphant.

Vostre estandart nous montrera l'adresse

Pour superer le tirant qui sans cesse

A vos barons faict inhumanité:

De les grever toujours s'est incité.

Quoy! Le souffrir? Ce vous seroit simplesse! (6 $\left.\mathrm{r}^{\circ}\right)$

Les variations dans l'écriture, qui peuvent passer de la prose curiale des translations de l'époque bourguignonne à un style alerte, ne dédaignant pas la grivoiserie, éventuellement associé au goût pour une écriture humanistique, cultivant les latinismes et les allusions mythologiques ${ }^{37}$, sont, il faut bien le constater, impuissantes à recréer la puissante alchimie de la chanson de geste ancienne: seule l'Italie parviendra, avec l'Arioste et ses successeurs, à recréer le miracle épique, à la fois en utilisant la forme poétique brève de l'ottava rima qui évite la déperdition d'énergie imputable aux trop longues laisses d'alexandrins, et en systématisant le mélange baroque des univers littéraires. Elle associe de la sorte, dans un tourbillon d'images et de références, les traditions mythologiques de l'Antiquité, le discours épique médiéval et l'univers arthurien.

43 Telles quelles pourtant, les proses épiques françaises ne sont pas prêtes, à la fin du $\mathrm{XV}^{\mathrm{e}}$ siècle, à disparaître de l'univers imaginaire des lecteurs, auxquels elles transmettent une sorte de trésor à la fois lointain et familier. En dépit du succès des romans d'aventures dont elles ne se distinguent guère, en dépit surtout de l'expansion fulgurante de nouveaux venus, comme les Amadis, qui, joints aux héros de l'Arioste et $\mathrm{du}$ Tasse popularisés par les traductions et d'innombrables adaptations lyriques, occupent tout l'horizon culturel, les éditions de Galien Restauré, des Conquestes du Gran Rot Charlemaine des Espaignes, des Quatre fils Aymon, de Huon de Bordeaux et de Valentin et Orson franchissent le barrage des siècles.

Les deux premiers textes font une place importante au récit épique de l'expédition d'Espagne et de la mort de Roland, dont le caractère essentiel a été reconnu à travers les âges: Galien à travers l'itinéraire guerrier et aventureux du fils d'olivier, les Conquestes - qui comportent aussi une biographie de Charlemagne -, à travers l'histoire de Fierabras et la tradition du Pseudo-Turpin. Le troisième texte est un récit de révolte, opposant à Charlemagne quatre fiers barons, aidés par l'enchanteur Maugis et le cheval Bayard; le quatrième présente avec le personnage d'Auberon et l'incorporation des suites de Huon d'importants éléments merveilleux et oppose à nouveau un baron à 
l'empereur. Le dernier accumule exploits guerriers et aventures nourries par des thèmes folkloriques, sur fond de lutte entre Chrétiens et Sarrasins. On peut considérer que ces récits, transmis par la librairie de colportage, sont destinés à faire œuvre de mémoire, présentant, à côté du Saint Alexis ou des arts de vivre divers, ce qu'il convient de retenir de l'histoire nationale. Valentin et Orson, dans le titre d'une édition Deckherr du XIX siècle, sont déclarés Fils de l'empereur de Grèce et neveux du très-vaillant et trèschrétien Pepin, roi de France ${ }^{38}$, tandis que l'Avis au lecteur, tiré lui aussi d'une édition Deckherr, de rédaction moderne mais affectant à plusieurs reprises un style archaïsant, insiste sur le caractère véridique de l'histoire des Quatre Fils Aymon:

Touchant la voie souterraine dudit château de Montauban, de laquelle parle le vingtième chapitre de ce livre, et par où se sauvèrent Regnault et les siens affamés par le long siège, c'est chose contenue en la vraie histoire de Froissard, $3^{e}$ volume, chap. 58, et dans le même est aussi parlé de l'antiquité de la maison de Regnault de Montauban ${ }^{39}$.

Tel est, rapidement esquissé, le cheminement de la tradition épique à partir du moment où s'est opéré le passage à la prose. Si l'on cherche à porter un diagnostic sur le phénomène, on pourra dire qu'il a servi sur la longue durée la mémoire de l'épopée, et qu'il a certainement contribué au mouvement de retour aux origines qui a conduit les érudits du XIX ${ }^{e}$ siècle à se tourner vers les textes-sources et à les éditer, sans que le courant des adaptations soit pour autant complètement interrompu.

Il a produit des récits variés, dont les plus intéressants sont ceux où une action complexe, abondant en situations dramatiques - les Quatre Fils Aymon notamment stimule l'attention du lecteur. Il n'a pas suscité, contrairement à l'Italie, de «sursaut poétique " capable de recréer, grâce à des formes nouvelles, l'association structurelle du lyrique et du narratif propre à la chanson de geste. Des essais dramatiques, qui mériteraient d'être étudiés, ont pourtant vu le jour, et l'on rappellera le succès extraordinaire de l'une de ces adaptations, La Fille de Roland, d'Henri de Bornier (1875) ${ }^{40}$, qui imagine de rassembler Ganelon, qui aurait survécu à la tragédie de Roncevaux et se dissimule sous le nom d'Amaury, Gérald son fils et Berthe, fille de Roland dans un drame de l'expiation, du pardon et du sacrifice. Gérald conquiert de haute lutte l'épée de Roland, jadis dérobée par un païen et renonce, quand il apprend sa véritable identité, à l'amour de Berthe :

«Je veux que le malheur soit plus grand que la faute!

Et le père sera d'autant mieux pardonné

Que le fils innocent se sera condamné !» (IV, 3)

47 Au goût du jour au moins, il n'est pas certain qu'un tel drame, ou plutôt mélodrame en alexandrins, trouve aujourd'hui encore des lecteurs: la Fille de Roland ne saurait rivaliser avec l'immense fresque du Soulier de satin de Claudel, qui atteint véritablement, sans parler de l'image filmique, la grandeur épique.

En ce qui concerne le récit en prose en tout cas, la vertu épique semble avoir quitté les rivages médiévaux, voire le registre guerrier : c'est, quel que soit le sujet traité, dans le surgissement et le chevauchement des images, dans le choc des sonorités, la sobriété et la puissance des dialogues, que continuent de se marier le narratif et le lyrique. Des textes aussi différents que ceux rassemblés par Éloïse Lievre dans son petit livre sur L'Épique $^{41}$ - la prise de la barricade dans les Misérables, l'agonie du Voreux dans Germinal, l'attente des condamnés à mort dans la Condition humaine - montrent bien que la prose peut être épique dans la fulgurance d'un passage, quel que soit le registre imaginaire et le contexte historique invoqués, plutôt que dans la continuité d'une matière ou d'un 
type de discours ${ }^{42}$. Dès lors, la prose épique ne peut plus prétendre à l'illustration d'une communauté nationale et d'une continuité généalogique déterminées: elle s'ouvre à l'universalité du temps et de l'espace ${ }^{43}$. Pourquoi ne pas s'en réjouir?

\section{NOTES}

1. Éd. M. Tyssens, N. Henrard et L. Gemenne, Paris, Champion, 2000, chap. 1, § 1, p. 1.

2. Voir l'édition Edmond A. Emplaincourt, The Old French Crusade cycle, vol. IX, The University of Alabama Press, 1989, p. 1.

3. Voir l'éd. R. Guiette, Bruxelles, 1940, p. 13.

4. Il s'agit du ms. Arsenal 3324 qui fait se succéder une version du Pseudo-Turpin et la mise en prose d'Anséis de Carthage.

5. $1 \mathrm{c}$.

6. Le texte répète « comme vent ».

7. Ibid.

8. Voir E. Emplaincourt, éd. cit., p. XIX-XX et E. Emplaincourt et Jan Nelson, « La Geste du Chevalier au Cygne : la version de Copenhague et la tradition du premier cycle de la croisade ", Romania 104, 1983, p. 351-370.

9. Éd. Giovanni Palumbo, Paris, Champion, CFMA 139, 2002. Voir aussi F. Suard, «Les Trois Fils de Roi : chronique d'une Europe réconciliée et victorieuse des Turcs », Par les mots et par les textes, Mélanges Claude Thomasset, Paris, PUPS, 2005, p. 729-745.

10. Éd. cit., p. 81.

11. Voir Alphonse Dupront, Le Mythe de la croisade, Paris, Gallimard, 4 vol., 1997.

12. Voir Maugis, ami de la fée Oriande, séduisant sa tante Gracienne, puis l'épouse de Marsile, ou bien les aventures amoureuses d'Ogier qui, dans les remaniements du XIV siècle, devient l'amant de la fée Morgue.

13. Éd. J.-Ch. Herbin, Prose des Loherains, Presses Universitaires de Valenciennes, 1995, p. 27.

14. Voir Garin le Loherenc, éd. A Iker-Gittleman, Paris, CFMA, 3 vol., 1996-1997, v. 9882-9986.

15. Voir David Aubert, Garin le Loherain, Publications de l'Université de Provence, 2005, p. 295-296, 1. 297-357.

16. Ces mises en garde figurent naturellement avant le départ de Bégon, v.960-611 pour la chanson et p. 289, 1. 51-59.

17. V. 9908-11.

18. La chanson en fait au contraire « i. bon serjant a pié » (v.9967).

19. À comparer avec les v. 9970-74 de la chanson.

20. Aubert note par exemple le fait que les chiens de Bégon flairent les arrivants ; «Et lorz qu'ilz l'eurent assez approchié et veu de si pres que bien les sentirent les chiens ", ed. cit., p. 295, 1. 303-304.

21. Galien le Restoré en prose, Paris, Champion, 1998, p. 35. Passage déjà cité dans notre article «Y a-t-il un avenir pour la tradition épique médiévale après 1400 ? ", CRM 11 spé, 2004, p. 84.

22. La Geste de Garin de Monglane en prose, éditée par Hans-Erich Keller, Aix-en-Provence, CUER MA, Senefiance $\mathrm{n}^{\circ} 35,1994, \mathrm{p} .185$.

23. Genève, Droz, TLF 536, 2001.

24. Éd. cit., p. 51-98. 
25. Éd. cit., p. 98.

26. Voir Le Huon de Bordeaulx en prose du XV siècle, Peter Lang, New York, 1998.

27. Éd. cit., p. clv.

28. Ces chiffres, comme les suivants, sont empruntés à P. Tylus, éd. cit., p. 91-92.

29. Le chapitre qui commence à la 1.4220 ne se termine qu'à la 1.4370 (150 l.); il ne comporte qu'un seul paragraphe. Le chapitre qui commence à la ligne 6716 comporte un seul paragraphe de 294 lignes (il se termine à la ligne 6999).

30. Voir de même, pour le chapitre qui commence à la ligne 4375 , l'incipit du chapitre et la 1. 4405.

31. Éd. cit., p. lx.

32. Éd. cit., 1. 1438-1445.

33. Éd. Hans-Erich Keller, L'Hystoire de Charlemagne, Genève, Droz, TLF 413, 1992, p. 102.

34. Ed. cit., chap. XVI, § 17, p. 173.

35. Voir notre article, «Le Beuves de Hantonne en prose : importance et expression du sentiment amoureux ", Chanson de geste et tradition épique en France au Moyen Âge, Caen, Paradigme, 1994, p. 399-414.

36. Éd. cit., chap. LIII, § 14, p. 554.

37. Voir notre article "Les libertés prises avec l'écriture des vieux romans au XVI ${ }^{\mathrm{e}}$ siècle ", Le Moyen Français, 51-53, 2002-2003, p. 530-546.

38. Lise Andries, Geneviève Bollème, La Bibliothèque Bleue, Paris, R. Laffont, col. Bouquins, 2003, p. 268.

39. Op. cit., p. 302. Le passage évoqué se trouve effectivement au livre III, §52, des Chroniques de Froissart, Paris, SHF, Champion, 1931, t. XII, p. 196. L'auteur de l'Avant-propos pouvait connaître les Chroniques par l'édition Dacier (1788) ou l'édition Buchon (1835).

40. Elle est traduite en suédois en 1876, en allemand en 1880 ; on l'édite encore en 1954.

41. Paris, Gallimard, La bibliothèque, 2002.

42. L'image filmique en revanche, lorsqu'elle résulte du travail d'un grand metteur en scène (Eisenstein, Kurosawa oui, tout récemment, Riddley Scott avec The Kingdom of Heaven), reste toujours susceptible, dans sa stylisation ou au contraire son foisonnement, de servir une action essentiellement guerrière dans un cadre historique médiéval.

43. Jean de Grouchy, on s'en souvient, vouait la chanson de geste ad conservandam civitatem (au salut de la communauté). Cela peut s'entendre d'une communauté étroite, comme le voulaitt sans doute le théoricien médiéval, mais aussi d'une communauté plus vaste, celle de l'humanité tout entière. 